\title{
Preconception Care Between Pregnancies: The Content of Internatal Care
}

\author{
Michael C. Lu • Milton Kotelchuck • \\ Jennifer F. Culhane • Calvin J. Hobel • \\ Lorraine V. Klerman · John M. Thorp Jr
}

Published online: 1 July 2006

(C) Springer Science+Business Media, Inc. 2006

\begin{abstract}
For more than two decades, prenatal care has been a cornerstone of our nation's strategy for improving pregnancy outcomes. In recent years, however, a growing recognition of the limits of prenatal care and the importance of maternal health before pregnancy has drawn increasing attention to preconception and internatal care. Internatal care refers to a package of healthcare and ancillary services provided to a woman and her family from the birth of one child to the birth of her next child. For healthy mothers, internatal care offers an opportunity for wellness promotion between pregnancies. For high-risk mothers, internatal care provides strategies for risk reduction before their next pregnancy. In this paper we begin to define the contents of internatal care. The core components of internatal care consist of risk assessment, health promotion, clinical and psychosocial interventions. We identified several priority areas, such as FINDS (family violence, infections, nutrition, depression, and stress) for risk assessment or BBEEFF (breastfeeding, back-to-sleep, exercise, exposures, family planning and folate) for health promotion.
\end{abstract}

\section{C. Lu ( $\square)$}

Department of Obstetrics and Gynecology,

David Geffen School of Medicine at UCLA,

Department of Community Health Sciences, Center for Healthier

Children, Families and Communities,

UCLA School of Public Health, Box 951772,

Los Angeles, CA 90095-1772

e-mail:mclu@ucla.edu

\section{Kotelchuck}

Department of Maternal and Child Health,

Boston University School of Public Health, Boston, MA

\section{J. F. Culhane}

Department of Obstetrics and Gynecology,

Drexel University College of Medicine, Department of Epidemiology and Statistics, Drexel School of Public Health, Philadelphia, PA
Women with chronic health conditions such as hypertension, diabetes, or weight problems should receive on-going care per clinical guidelines for their evaluation, treatment, and follow-up during the internatal period. For women with prior adverse outcomes such as preterm delivery, we propose an internatal care model based on known etiologic pathways, with the goal of preventing recurrence by addressing these biobehavioral pathways prior to the next pregnancy. We suggest enhancing service integration for women and families, including possibly care coordination and home visitation for selected high-risk women. The primary aim of this paper is to start a dialogue on the content of internatal care.

Keywords Preconception care - Internatal care · Content . Preterm birth · Interpregnancy

For more than two decades, prenatal care has been a cornerstone of our nation's strategy for improving pregnancy outcomes. In recent years, however, the effectiveness of this strategy has been called into question [1]. Increasing use of

\footnotetext{
C. J. Hobel

Cedars-Sinai Medical Center and Burns Allen Research Institute,

Department of Obstetrics and Gynecology,

David Geffen School of Medicine at UCLA,

Los Angeles, CA

L. V. Klerman

The Heller School for Social Policy and Management,

Brandeis University,

Waltham, MA

J. M. Thorp Jr

Department of Obstetrics and Gynecology,

University of North Carolina-Chapel Hill,

Chapel Hill, NC
} 
early and adequate prenatal care has not led to a significant decline in low birthweight (LBW) or prematurity, which are leading causes of infant mortality in the U.S.. Nor has closing the access gap in prenatal care led to a significant reduction in racial-ethnic or socioeconomic disparities in these adverse birth outcomes. There is now a growing consensus that further reduction in this nation's LBW and prematurity rates cannot be achieved solely by improving access to prenatal care [2].

A limitation on the effectiveness of prenatal care may be its timing. Many of the pathophysiological pathways leading up to adverse birth outcomes may have their onset early in pregnancy, possibly at or even before implantation. By the time prenatal care is initiated, it may be too little too late to significantly alter the course or outcome of the pregnancy. Indeed, there is now a growing recognition that birth outcomes are the end product of not only the nine months of pregnancy, but the entire life course of the mother leading up to the pregnancy [3]. Improving birth outcomes, therefore, takes promoting maternal health not only during pregnancy, but before and between pregnancies and, indeed, across the woman's lifespan.

The recognition of the importance of maternal health before pregnancy has led to a growing interest in preconception care [4]. A special subtype of preconception care is internatal care. Internatal care refers to a package of healthcare and ancillary services provided to a woman and her family from the birth of one child to the birth of her next child. It is, in essence, preconception and prenatal care for a subsequent pregnancy. We prefer the term "internatal care" over the more popular term "interconception care" (from the conception of one pregnancy to the conception of the next pregnancy) because 1) internatal care represents an extension of prenatal care with which the public is familiar; 2) the point of entry into care is the birth of one child; and 3) the endpoint of care is the birth of the next child. And while in this paper we will focus primarily on the interpregnancy interval (from the termination of one pregnancy to the conception of the next pregnancy), we prefer the term "internatal care" over "interpregnancy care" because internatal care suggests a continuity of care into a subsequent pregnancy.

Despite the growing interest, for most people it remains unclear what is meant by internatal care. For example, many Healthy Start programs now offer internatal care, and yet the content of care varies greatly across sites. There have also been a few intervention studies on internatal care, most notably in Denver [5], Atlanta [6], and Philadelphia [7], with vastly different approaches. The lack of some standards of care makes it difficult to move forward research, practice, and policy, or even discussion, on internatal care. The purpose of this paper is to begin to define the content of internatal care.
For healthy mothers, internatal care offers an opportunity for wellness promotion between pregnancies. Presently many women lack such opportunity, particularly among lowincome and minority women. In most states, Medicaid programs terminate pregnancy-related healthcare coverage for most low-income mothers at 60 days postpartum. Other than the one recommended postpartum visit, many of these women will have little or no access to healthcare between pregnancies. Internatal care can help close the gap in healthcare for these women. Even for women with healthcare coverage, it is unclear how much and what internatal care they are currently getting. In this paper, we begin to define the core contents of universal internatal care that all women of childbearing age should receive between pregnancies.

While we believe that internatal care should be universally available to all women between pregnancies, it may be particularly beneficial for high-risk mothers, such as women with chronic diseases or prior adverse pregnancy outcomes. Many such outcomes (e.g. prematurity, fetal death) carry a high recurrence risk in a subsequent pregnancy, and the biobehavioral risk factors are often carried from one pregnancy to the next [8]. For these mothers, internatal care offers an opportunity for risk reduction before their next pregnancy. In this paper, we also begin to describe the content of enhanced internatal care for high-risk mothers. We will use women with chronic health conditions (hypertension, diabetes, or weight problems) and women with a prior preterm delivery as examples of what more can be done for high-risk mothers during internatal care than under current prevailing practice which, for many women, consists of the one recommended six-week postpartum visit, or none at all.

Two decades ago, the U.S. Public Health Service assembled an expert panel to define the contents of prenatal care. In its report Caring for Our Future: The Content of Prenatal Care, the Expert Panel identified four basic components of prenatal care consisting of 1) early and continuing risk assessment, 2) health promotion, 3) clinical interventions, and 4) psychosocial interventions [9]. We now use the same framework to outline the contents of internatal care, based on our search of the literature for clinical standards, best practices, and proven or promising strategies. The focus of this paper is on the content of internatal care, particularly during clinical visits. Other important topics, such as financing, organization, delivery, motivation, and community interventions, are beyond the scope of this paper and will be addressed in a series of papers to follow.

It is not our intention in this paper to propose a complete and final model of internatal care. Instead, we present here the start of a framework for internatal care, to be filled in through more dialogues and consensus-building which we hope this paper will help move forward. 


\section{Recommended core contents of universal internatal care for all women following a pregnancy}

Most pregnant women in the United States are healthy and experience healthy pregnancy and birth outcomes. For these women, internatal care is still important because it offers an opportunity for wellness promotion. In this section, we describe the core contents of internatal care, including risk assessment, health promotion, clinical interventions, and psychosocial interventions (Table 1). These components should be offered to every woman following a pregnancy, regardless of her risk status. We recommend expanding the current six-week postpartum visit to three or more internatal visits, at 2 weeks, 6 weeks, and 6 months postpartum, with annual follow-up beginning at one year postpartum. We will expand on the rationale for the recommended schedule of visits, and address issues related to systems integration later in this section.

\section{Risk assessment}

The primary objective of risk assessment is to identify ongoing problems that need to be addressed. Most pregnant women in the U.S. are healthy and thus should have no major problems postpartum. However, some medical, behavioral or psychosocial problems may have been overlooked by their prenatal care providers. Other problems may develop during the internatal period. Providers of internatal care should pay particular attention to five problems that are now commonly missed during prenatal or postpartum care: family violence, infection/immunization, nutrition, depression and stress (FINDS).

\section{Family violence}

The experience of family violence may be quite common among pregnant and postpartum women in the U.S. Most studies of the prevalence of physical abuse during pregnancy report estimates in the range of 4 to 8 percent, though higher estimates (around 20\%) have been reported in some populations [10]. Less is known about the prevalence of violence after pregnancy, with estimates at 3 months postpartum ranging from $3.2 \%$ in a population-based survey to $21 \%$ in a prospective study of adolescent mothers $[11,12]$. In the latter study [12], three of four women who reported intimate partner violence (IPV) had not reported IPV prior to delivery. Family violence puts maternal health and child development at great risk. The literature contains solid links between intimate partner violence and child abuse [13]. Because violence is frequently missed by healthcare providers including prenatal care providers, we recommend routine screening for family violence during every internatal visit. Several brief screening instruments, such as the 4-item Hurt Insulted Threatened or Screamed at (HITS), the 3-item Partner Violence Screen (PVS), and a 3-item Abuse Assessment Screen (AAS), can be effectively used to screen for IPV in a clinical setting [13].

\section{Infection/immunization}

Some infections may go unrecognized or untreated during pregnancy. Chronic, untreated infections, such as periodontal disease, could pose a threat not only to the next pregnancy [14], but also to the mother's long-term health as well [15]. Most women are not screened for periodontal disease during prenatal care, and many women lack access to oral health services following pregnancy. While presently there is insufficient evidence to justify universal screening for asymptomatic infections among "low-risk" women in the internatal period (other than the U.S. Preventive Services Task Force (USPSTF) recommendation for Chlamydia screening in women under the age of 25) [16], in some populations routine screening for periodontal, sexuallytransmitted and some urogenital tract infections may be warranted.

Internatal care also offers an important opportunity to update women's immunization status. The USPSTF and other national advisory committees recommend that 1 ) all adults receive periodic diptheria-tetanus toxoids booster every 10 years, 2) all young adults receive a series of Hepatitis B vaccines, 3) all adults born after 1956 who lack evidence of immunity receive vaccination against measles and mumps, and 4) all women of childbearing age be screened for rubella and varicella susceptibility; susceptible nonpregnant women of childbaring age should be offered vaccination and avoid conception for at least 28 days after vaccination; susceptible pregnant women should be vaccinated immediately after delivery (Table 1). Immunization status should be assessed and updated at the six-week internatal visit.

\section{Nutrition}

Nutrition can play an important role in promoting maternal health and child development, and yet women's nutritional needs are often overlooked by prenatal or postpartum care providers. One recent population-based survey in California found that nearly one in three pregnant women who were income-eligible for Women, Infants, and Children (WIC) program reported being food-insecure; of these, one in four reported not being enrolled in WIC [17]. Nutritional screening should address the ABCD's of nutritional risks: 
Table 1. Recommended content and schedule of internatal care, and organizations recommending these practice standards

\begin{tabular}{|c|c|c|}
\hline Risk assessment & Organizations $^{a}$ & Schedule ${ }^{b}$ \\
\hline \multicolumn{3}{|l|}{ Core content of internatal care for all women } \\
\hline Family violence & JACHO, AMA & $1,2,3$ \\
\hline \multicolumn{3}{|l|}{ Infections } \\
\hline Chlamydia & USPSTF, ACPM & 2 \\
\hline Periodontal & ADA & 2 \\
\hline \multicolumn{3}{|l|}{ Immunizations } \\
\hline Diptheria-tetanus booster & USPTSF, ACPM, HHS & 2 \\
\hline Hepatitis B & USPTSF, ACPM, HHS & 2 \\
\hline Measles and mumps & USPTSF, ACPM, HHS, ACIP & 2 \\
\hline Rubella & USPTSF, ACPM, HHS, ACIP & 2 \\
\hline Varicella & USPTSF, ACPM, HHS, ACIP & 2 \\
\hline Nutrition & USPSTF & $1,2,3$ \\
\hline Depression & USPSTF, ACOG & $1,2,3$ \\
\hline Stress & & $1,2,3$ \\
\hline \multicolumn{3}{|l|}{ Health promotion } \\
\hline Breastfeeding & AAP, HP2010 & $1,2,3$ \\
\hline Back-to-Sleep & AAP, NICHD, HP2010 & 2 \\
\hline Exercise & NHLBI, HHS, HP2010 & 2 \\
\hline \multicolumn{3}{|l|}{ Exposures } \\
\hline Smoking, alcohol, drug use & HHS, ACOG & $1,2,3$ \\
\hline Mercury & FDA/EPA & 2,3 \\
\hline Lead & $\mathrm{CDC}, \mathrm{AAP}$ & 2,3 \\
\hline Dioxins & IOM & 2,3 \\
\hline Indoor/outdoor pollutants \& allergens & NHLBI & 2,3 \\
\hline Family planning & ACOG/AAP & $1,2,3$ \\
\hline Folate supplementation & $\mathrm{CDC}, \mathrm{AAP}$ & 3 \\
\hline \multicolumn{3}{|l|}{ Clinical interventions } \\
\hline Height and weight measurements & ICSI, ACOG & $1,2,3$ \\
\hline Blood pressure & ICSI, ACOG & 2 \\
\hline Total skin examination & ACS, ACPM & 2 \\
\hline Clinical breast examination & ACS, ACOG & 3 \\
\hline Papanicolau smear and pelvic examination & ACS, ACOG & 2 \\
\hline Screening mammography & ACS, ACOG & 3 \\
\hline \multicolumn{3}{|l|}{ Psychosocial interventions } \\
\hline Social services & & $1,2,3$ \\
\hline Clinical support & & $1,2,3$ \\
\hline Parenting support & & $1,2,3$ \\
\hline \multicolumn{3}{|l|}{ Enhanced content of internatal care for high-risk women } \\
\hline \multicolumn{3}{|l|}{ Chronic hypertension } \\
\hline Risk assessment, health promotion, clinical \& psychosocial interventions & NHLBI & \\
\hline \multicolumn{3}{|l|}{ Gestational hypertension } \\
\hline Risk assessment, health promotion, clinical \& psychosocial interventions & NHLBI, ACOG & \\
\hline \multicolumn{3}{|l|}{ Pregestational diabetes } \\
\hline Risk assessment, health promotion, clinical \& psychosocial interventions & ADA & \\
\hline \multicolumn{3}{|l|}{ Gestatational diabetes } \\
\hline Risk assessment, health promotion, clinical \& psychosocial interventions & ADA, ACOG & \\
\hline \multicolumn{3}{|l|}{ Overweight/obesity } \\
\hline Risk assessment, health promotion, clinical \& psychosocial interventions & NHLBI, HHS USPSTF & \\
\hline \multicolumn{3}{|l|}{ Preterm birth } \\
\hline \multicolumn{3}{|l|}{ Risk assessment } \\
\hline Reproductive history & ACOG & \\
\hline Family history & ACOG & \\
\hline Medical assessment & ACOG & \\
\hline Nutritional assessment & $\mathrm{ACOG}$ & \\
\hline Social assessment & ACOG & \\
\hline
\end{tabular}


Table 1. Continued

\begin{tabular}{ll}
\hline Risk assessment & Organizations $^{a}$ \\
\hline Health Promotion & \\
Smoking cessation & HHS \\
Substance abuse treatment & ACOG \\
Optimal prepregnancy BMI & IOM \\
Ensure adequate omega-3 fatty acids intake & ISSFAL, IOM \\
Family planning & ACOG/AAP \\
Clinical interventions & \\
Progesterone use in subsequent pregnancy & ACOG \\
Psychosocial Interventions & \\
Services for children with special health care needs & AAP \\
Home visitation & AAP \\
\hline
\end{tabular}

${ }^{a}$ References to these recommendations available through the authors. Abbreviations: JACHO, Joint Commission on Accreditation of Healthcare Organizations; AMA, American Medical Association; USPSTF, United States Preventive Services Task Force; ACPM, American College of Preventive Medicine; ADA, American Dental Association; HHS, Department of Health and Human Services; ACIP, Advisory Committee on Immunization Practices (CDC). ACOG, American College of Obstetricians and Gynecologists; AAP, American Academy of Pediatrics; HP2010, Healthy People 2010; FDA, Food and Drug Administration; EPA, Environmental Protection Agency; CDC, Centers for Disease Control and Prevention; IOM, Institute of Medicine; NHLBI, National Heart, Lungs, and Blood Institute; ICSI, Institute for Clinical Systems Improvement; ACS, American Cancer Society; ADA, American Diabetes Association.

${ }^{b}$ Recommended schedule for each component of core content of internatal care; $1=2$-week visit, $2=6$-week visit; $3=6$-month visit . Schedule for enhanced content varies according to need

anthropometric (e.g. low or high body mass index), biochemical (e.g. for anemia or folate deficiency in some populations), clinical (e.g. eating disorder) and dietary (e.g. content, pattern, food insecurity). An abbreviated 6-item version of the Household Food Security Scale can be used to screen for food insecurity. Inquiry should be made about folate or multivitamin supplementation (see under Health Promotion). We recommend routine screening of nutritional risks at every internatal visit.

\section{Depression}

Maternal depression affects a large number of women and their children. A recent systematic review reported combined estimates of the point prevalence of major and minor depression ranging from 8.5 to 11 percent during pregnancy, and 6.5 to nearly 13 percent during the first year postpartum [18]. Maternal depression can have negative long-term impact on both maternal health and child development, but is often missed by prenatal and postpartum care providers. We recommend routine screening for maternal depression at every internatal visit. Fairly accurate and feasible screening measures are available, including the Postpartum Depression Screening Scale (PDSS), Edinburgh Postnatal Depression Scale (EPDS), Beck Depression Inventory (BDI), and the Center for Epidemiologic Studies Depression Scale (CESD) [18]. The evidence available, albeit limited, suggests that providing some form of psychosocial support to pregnant and postpartum women at risk of having a depressive illness may decrease depressive symptoms [18].

\section{Stress}

Maternal stress is perhaps the hardest to screen; clinicians are often at a loss as to how to screen or what to do with a positive screen. Yet chronic stress can pose significant risks to maternal health and child development. For the mother, chronic stress can cause wear and tear to her body's adaptive systems, what McEwen refers to as allostatic load, which could lead to more rapid deterioration in her health and function over time [19]. For the infant (or the fetus in a subsequent pregnancy), maternal stress could result in the re-programming of the child's basic autonomic rheostat and alteration of immune regulation, leading to increased vulnerability to disease and dysfunction later in life [20]. There is an important need for the development of a multi-dimensional screening tool for stress that can be feasibly implemented in a clinical setting. For now, clinicians need to ask about major stressors in women's lives (e.g. homelessness, unemployment, family violence, social isolation) at every internatal visit, and find resources and supports to help the family deal with these stressors.

Thus even for healthy mothers with healthy infants, much can be done to reduce health risks and promote well-being during internatal care. In addition to the five areas we highlighted (FINDS-family violence, infections/immunization, nutrition, depression, and stress), a battery of reproductive, medical, family, genetic and psychosocial risks should be routinely assessed during internatal care. Other areas of risk assessment can be added to the contents of internatal care as the model becomes more fully developed. 


\section{A word of caution about risk screening}

Presently a major limitation on the effectiveness of risk screening is the lack of available or accessible services for those with a positive screen. The benefit of depression screening is limited when there are no mental health services available or accessible to refer mothers who screen positive for depression. And a lack of support services (and coordination thereof) for abuse victims is identified by many providers as a major deterrent to screening for family violence. For risk screening to be effective, it needs to be followed up by effective interventions. This means having all the necessary resources and service capacity (e.g. oral health services for those with periodontal disease, nutritional support for those who are food-insecure, housing assistance for homeless mothers and families) to assist those with a positive screen.

\section{Health promotion}

The primary objective of health promotion is to promote the health and wellbeing of the mother, infant, and family. We will highlight six components health promotion during internatal care: breastfeeding, back-to-sleep, exercise, exposures, family planning and folic acid (BBEEFF).

\section{Breastfeeding}

Despite well-known benefits of breastfeeding for both mother and infant [21], at least one-third of mothers in the U.S. never initiate breastfeeding [22]. Among women who initiated, nearly one in five will stop nursing by one month, and nearly two-thirds will stop by six months [22], despite current recommendation to breastfeed for at least one full year [21]. Internatal care offers an important opportunity to promote breastfeeding, particularly the initial two-week visit to address nascent problems, the six-week visit to anticipate problems related to transition back to work, and the six-month visit to encourage continued breastfeeding with introduction of solid foods.

\section{Back-to-sleep}

With the introduction of the Back-to-Sleep campaign by the American Academy of Pediatrics in 1992, the overall incidence of sudden infant death syndrome (SIDS) has decreased by almost 50\% [23]. Despite this success, SIDS continues to be a leading cause of infant death in the U.S., as well as racial disparity in infant mortality [23]. SIDS rate among black infants remains more than twice that of white infants, even after the implementation of the Back-to-Sleep campaign [23]. Black infants are more likely than white infants to be placed in the prone position and soft sleep surfaces for sleep [23]. Providers of internatal care need to discuss the infant's sleep environment with the parents. Because the risk of SIDS peaks at 2 to 4 months of age, the six-week visit offers an important opportunity to talk about back-tosleep.

\section{Exercise}

Health education about physical activities should be part of an on-going effort for wellness promotion during internatal care. The 1988 National Maternal and Infant Health Survey revealed that 25 percent of white women and 45 percent of black women were heavier by $4.1 \mathrm{~kg}$ (nearly 9 pounds) or more at 10 to 18 months post partum than they were before pregnancy [24]. Clinicians can help women develop a weight loss program that consists of healthy dieting, physical activities, and behavioral modifications if needed. The National Heart, Lung, and Blood Institute recommends that all adults should set a long-term goal to accumulate at least $30 \mathrm{~min}$ or more of moderate-intensity physical activity on most, and preferably all, days of the week [25]. For women in the postpartum period, prepregnancy exercise routines may be resumed gradually as soon as it is physically and medically safe. Clinicians need to routinely discuss physical activity with the woman, particularly at the six-week and six-month visits.

\section{Exposures}

Cigarette smoking poses a significant threat to the health of the mother, her infant, and her subsequent pregnancy. Approximately one in eight (11.2\%) U.S. women who gave birth in 2002 reported smoking cigarettes during pregnancy [26]. Furthermore, up to $70 \%$ of women who quit smoking during pregnancy will relapse within 6 months of delivery [27]. Effective models of clinical intervention for smoking cessation (e.g. 5A's) and relapse prevention have been developed and should be incorporated into internatal care [28]. Prenatal exposure to alcohol and other substances like cocaine and amphetamines have been associated with birth defects as well as adverse pregnancy and neurodevelopmental outcomes. According to the Behavioral Risk Factor Surveillance System, in 1999 the prevalence of any alcohol use and binge drinking among pregnant women in the U.S. was 12.8 and $2.7 \%$, respectively [29]. Postpartum use and recidivism rates are less well described. Several screening questionnaires have been developed to detect problem drinking which may also prove helpful in detecting substance abuse, include T-ACE (tolerance, annoyed, cut down, eye opener) questions, the CAGE (cut-down, annoyed, guilty, eye-opener) questionnaire, and the brief MAST questionnaire [30]. All women should be screened at the time of their first internatal visit, and referral for evaluation and treatment should be offered to those who 
screen positive. Additionally, mothers whose infants are at risk for neurobehavioral problems from prenatal exposures should be provided resources for early developmental evaluation and interventions for their infants [30].

A large number of environmental exposures during the internatal period can affect maternal health and child development. For example, chronic exposures to indoor and outdoor pollutants and allergens, including household molds and dust mites, may increase the child's future risk for atopy, allergies, and asthma. Less is known about the long-term effects of such exposures to maternal health and subsequent pregnancy outcomes. Lead is a potent developmental neurotoxicant and immunotoxicant, and household exposures can come from old paint, leaching from plumbing, and ceramic dinnerware and glazes. Certain measures can be taken to reduce household exposures to pollutants, allergens and lead. Exposure to mercury can be reduced by adherence to the EPA/FDA joint advisory related to consumption of fish and shellfish, and exposure to dioxins can be reduced by decreasing dietary fat consumption (Table 1). Clinicians need to assess and discuss with their patients avoidance of exposures that could adversely impact on maternal health and child development at every internatal visit, but particularly at the 6-week and 6-month visits.

\section{Family planning}

Family planning is vital to the health and wellbeing of women and their families [31]. Unintended pregnancy and short interpregnancy intervals are associated with increased risk for adverse birth outcomes [31, 32]; family planning can reduce the risk by promoting effective contraceptive use and optimal birth spacing. An unintended pregnancy also puts future maternal health and social wellbeing at risk; family planning can protect maternal health and choice [31]. Discussion of family planning should begin prenatally and prior to discharge from the hospital; it should be addressed again at the two-week and six-week internatal visits, including risks, benefits and side effects of available contraceptive options and plans for future childbearing. Adherence to method and side effects should be reassessed at the six-month visit.

\section{Folate supplementation}

Folate is important to both maternal and child health. Maternal folate deficiency has been linked to future risk for cardiovascular and other chronic adult diseases, as well as neural tube and other birth defects in the offspring. Periconceptional (preconceptional and in early pregnancy) use of folic acid has been demonstrated to reduce the occurrence and recurrence of neural tube defects [33]. Thus the U.S. Public Health Services recommends that all women of childbearing age consume 400 micrograms of folic acid daily [34].
Even though there are several ways of getting this amount of folic acid everyday, two-thirds of U.S. women of childbearing age still do not consume enough folic acid, and surveys continue to find substantial knowledge gaps regarding the benefits of folic acid. Folic acid should be promoted at every internatal visit, or at the minimum during the six-month visit. We feel that the recommendation for folic acid can be broadened to include daily multivitamin supplementation given the potential benefits of a number of other vitamins (e.g. B12) and elements to maternal health and subsequent pregnancy outcomes, particularly for women with nutritional deficiencies.

Table 1 summarizes the core contents of health promotion during internatal care, with an emphasis on BBEEFF (breastfeeding, back-to-sleep, exercise, exposures, family planning and folic acid). Other topics can be added to health promotion as the model of internatal care becomes more fully developed.

\section{Clinical interventions}

Most women in this category are, by definition, healthy and require little in the way of clinical interventions during internatal care, other than the standard preventive health services recommended for all women of childbearing age (Table 1). These include periodic height, weight and blood pressure measurements, total skin examination, clinical breast examination, Pap smear and bimanual pelvic examination every 1 to 3 years. Mammography should also be performed in women ages 40 and above. We will address clinical interventions for women with chronic conditions in the next section.

\section{Psychosocial interventions}

Many healthy women who gave birth to a healthy infant will nonetheless need some psychosocial support. There are three types of social support that need to be made available as part of the core package of internatal care services: 1) social services, 2) clinical support, and 3) parenting support (Table 1). Social services may include assistance with obtaining certain public insurance (e.g. SCHIP) or benefits (e.g. TANF, EITC). Some women may not be aware of their eligibility for child care subsidies (e.g. CCDF) or availability of early childhood education programs. Women experiencing intimate partner violence may need multiple medical, legal, and social services and could use some help with service coordination. The internatal visits provide a platform for accessing these social services.

Women who experience postpartum depression or other affective disorders may benefit from some forms of psychological support and therapy during the internatal period. Couples who experience problems with marital or sexual 
relationship in the internatal period may benefit from counseling or interventions. Women with alcohol or drug problems could also use clinical support and treatment. These and other professional clinical support services should be made available and accessible as part of standard internatal care services.

Many parents could benefit from some parenting support, such as mother support groups or parenting classes. A current innovative idea in prenatal care is "centering" in pregnancy, which provides social support to pregnant women through group prenatal care [35]. While this idea remains to be tested, we believe that group internatal care-a "centering" in internatal care model-may provide additional support for some women, particularly around issues related to parenting practices and the stress of parenting.

\section{A note on psychosocial interventions}

Throughout this paper, the readers will notice an imbalance in the levels of details we used to describe clinical and psychosocial interventions. That is, clinical recommendations are, for the most part, described with much greater specificity than psychosocial interventions. It is certainly not our intention to propose a predominantly biomedical model of internatal care. Rather, the lack of specificity in our description of psychosocial interventions reflect both the complexity of psychosocial issues that families-particularly low-income families-face for which there are often no easy solutions, as well as the dearth of well-designed and evaluated intervention programs addressing these psychosocial issues. For example, mental health services alone are unlikely to be effective in treating the postpartum depression of a homeless mother, and housing assistance alone is unlikely to find her stable housing without sustainable income and employment, but few well-designed intervention studies have critically evaluated the impact of a comprehensive package of mental health services, housing assistance, work- and life-skills training and childcare on maternal and family health. Our model of internatal care is unlikely to be effective without effective psychosocial interventions, but we will not know what these are as long as we keep looking for quick biomedical fixes. Future intervention studies on internatal care need to take a more integrative, comprehensive approach to improving family and women's health.

Service coordination and integration

As aforementioned, presently some referral services (e.g. mental health services, oral health services) are unavailable or inaccessible to many women between pregnancies. But even if the services are available, they are often poorly coordinated. Fragmentation in service delivery can deter access to care, particularly for low-income women with many other competing needs. Providers of internatal care need to consider how to better coordinate and integrate services in order to improve access. This can be accomplished by establishing a well-developed referral network. In some populations, care coordination or case management provided by a nurse or social worker may be needed. Ideally these services should be provided at one location to increase service coordination and integration. For example, the two-week internatal visit can be provided at the same time and location as the two-week well-baby check-up, with on-site WIC, health education, and social services that allow for "one-stop shopping." We will take up issues related to the organization and delivery of internatal care that will maximize access and utilization of services in a series of papers to follow.

\section{Schedule of visits}

In developing a schedule of visits for internatal care, we had to first wrestle with two questions. First, shouldn't the schedule of visits be individualized according to individual needs? While we support this view, we nonetheless feel that it may be useful to recommend some minimum standards to start a dialogue about this topic. For healthy women with healthy infants, we propose expanding the current sixweek postpartum visit to three or more internatal visits, at 2 weeks, 6 weeks, and 6 months postpartum, with annual exams beginning at one year postpartum. For some activities, the six-week postpartum visit may be too little too late. For example, one in five mothers who initiated breastfeeding will quit nursing within the first month [22]; a 2-week postpartum visit may offer more timely encouragement and interventions for maintenance of breastfeeding than a 6-week visit could. The 2-week visit also offers an opportunity to follow-up on wound care for cesarean delivery. Whenever possible, this visit should be coordinated with the two-week well-baby check-up, preferably at the same location, to increase motivation for attendance. We feel that it is important to keep the current six-week postpartum visit as the second internatal visit. This is a well-established visit in obstetrics, with well-defined contents. This visit, however, can be made more useful by expanding its contents. We feel that a third internatal visit at 6 months is important for follow-up on a number of issues, such as breastfeeding and family planning. The standard annual visits should begin at one year postpartum (Table 1).

Second, shouldn't any routine visit by a woman who may, at some time, become pregnant again be viewed as an opportunity to emphasize the importance of internatal health and habits [36]? While we support this view, we nonetheless feel that it would be preferable to recommend a schedule of internatal visits rather than relying on "any routine visit." Several components of internatal care are time sensitive (e.g. counseling about breastfeeding and family planning at 
2 weeks or about SIDS at 6 weeks) which should not wait for any routine visit. Scheduled internatal visits may be particularly important for women who are not making routine visits, or who are seeking care through the emergency department or urgent care clinics which lack the resources and capacity to provide comprehensive internatal care. We would also like to see internatal care become reimbursable visits, with standardized contents and schedule (rather than as an add-on to a visit for mastitis or cystitis), which may increase provider incentives to provide internatal care. Issues related to patient motivation and provider reimbursement will be addressed in a series of papers to follow.

\section{Recommended contents of enhanced internatal care for high-risk women}

In this section, we begin to define the contents of enhanced internatal care for high-risk women (Table 1). We focus on four groups of high-risk women: 1) women with chronic hypertension or history of hypertensive disorders during pregnancy, 2) women with pregestaional or gestational diabetes mellitus, 3) women who are underweight, overweight or obese, and 4) women with prior preterm birth. We call attention to the first 3 groups because they are often underserved in the internatal period, especially if they gave birth to a healthy infant. Their continued health needs may not be met because they had a good birth outcome. Some women will lose healthcare coverage (e.g. pregnancy-related Medicaid), and will have little on-going care despite their chronic conditions. We call attention to the 4th group because of their high recurrence risk [8] and the significance of recurrence. The goals of internatal care for these women are to provide ongoing care for their health problems and optimize their health prior to next pregnancy. In addition to the core contents of universal internatal care recommended above for all women of childbearing ages, we will examine what enhanced contents may benefit their long-term health, as well as the outcome of their subsequent pregnancy.

Women with chronic hypertension or hypertensive disorders during pregnancy

Hypertensive disorders are the most frequently reported medical risk factor during pregnancy [11]. Among the 4 million U.S. women who gave a live birth in 2002, more than 150,000 had "pregnancy-associated" (gestational) hypertension, more than 3,000 had chronic hypertension, and nearly 13,000 had eclampsia [11].

Among women with chronic hypertension (i.e. hypertension diagnosed prior to twentieth week of gestation and probably antedates the pregnancy) that persists after pregnancy, guidelines for evaluation and treatment are well es- tablished and can be incorporated into the contents of internatal care [37]. Risk assessment has three major objectives: 1) to assess lifestyle and identify other cardiovascular risk factors or concomitant disorders that may affect prognosis and guide treatment 2) to reveal identifiable causes of hypertension, and 3) to assess the presence or absence of target organ damage and cardiovascular disease. Initial evaluation should include medical history, physical examination (including fundoscopic and cardiovascular examinations), laboratory testing and other diagnostic procedures (including an electrocardiogram and testing for renal function and lipoprotein profile). Health promotion should promote weight reduction, DASH (Dietary Approaches to Stop Hypertension) eating plan, dietary sodium reduction, physical activities, moderation of alcohol, and smoking cessation. Clinical interventions should include drug therapy for all women with hypertension, as well as prehypertensive women with compelling indications (e.g. chronic kidney disease or diabetes). Follow-up should be monthly until the blood pressure goal is achieved, and then at 3-6 months interval thereafter during the internatal period. Psychosocial interventions should address chronic stressors that may exacerbate the chronic hypertension, as well as barriers (e.g. financial, motivational) to adherence with treatment protocol.

The contents of internatal care for women with gestational hypertension, pre-eclampsia, and eclampsia are less well defined, especially if the hypertension does not persist following pregnancy. In most women with gestational hypertension, the blood pressure will become normal during the first week postpartum; for women with preeclampsia the hypertension will take longer to resolve. If the hypertension has not remitted by the 6-week visit, the women should be reexamined 6 weeks later, when persisting pathology will probably be chronic [38]. During internatal care, providers need to discuss with their patients recurrence risk in the subsequent pregnancy. They also need to be aware that preeclampsia has been associated with increased risk of maternal cardiovascular diseases later in life [39], and that attention to cardiovascular risk factors is required in all subsequent internatal care and wellness visits. Presently there is no proven internatal clinical intervention for the prevention of recurrent gestational hypertension or preeclampsia.

During a subsequent pregnancy, antihypertensive therapy should be used only for pregnant women with severe hypertension, as pharmocologic treatment of women with uncomplicated mild chronic hypertension has not been found to be beneficial [38]. Several studies failed to demonstrate a benefit for low-dose aspirin in preventing preeclampsia among women with chronic hypertension [38]. When chronic hypertension is complicated by intrauterine growth restriction or preeclampsia, fetal surveillance is warranted. For women with preeclampsia during a previous pregnancy, low-dose 
aspirin started in the second trimester of pregnancy did not reduce the recurrence risk of preeclampsia [38]. Further studies are needed to identify effective interventions during the internatal period and subsequent pregnancy to prevent recurrent gestational hypertension or preeclampsia.

Women with pre-gestational or gestational diabetes mellitus

Diabetes complicating pregnancy is the second most frequently reported medical risk factor during pregnancy [11]. In 2002, more than 130,000 women reported having diabetes during pregnancy [11]. For women with pre-gestational diabetes, they are at increased risk for microvascular, neuropathic and cardiovascular diseases, especially if the diabetes is long-standing and poorly controlled. Their risks are reduced with tight glycemic control. Additionally, their fetuses are at increased risk for stillbirth and congenital anomalies. Preconception glycemic control among women with pre-gestational diabetes has been shown to reduce the risk of congenital anomalies [40]. Women who had gestational diabetes have $30-70 \%$ chance of recurrence during their next pregnancy [41] and an increased risk of developing Type II diabetes later in life [42]. Gestational diabetes increases the risk of fetal macrosomia, birth trauma, newborn hypoglycemia and hyperbilirubinemia. Evidence from animal models suggests that gestational diabetes, especially if poorly controlled, may also program insulin and leptin resistance in the fetus, leading the greater susceptibility for diabetes and obesity in the offspring [43].

For women with pre-gestational diabetes, internatal care should follow the guidelines issued by the American Diabetes Association on preconception care of women with chronic diabetes [44]. At the initial office visit, risk assessment should include a complete history, physical examination (including cardiovascular, neurological, and dilated retinal exams), and laboratory evaluation (including glycosylated hemoglobin (HbA1c), serum creatinine and 24-h urinary excretion of total protein and/or albumin, and measurement of thyroid stimulating hormone and/or free thyroxine level in women with type 1 diabetes because of the 5 to $10 \%$ coincidence of hyper- or hypothyroidism) and electrocardiogram if the diabetes has been long-standing. Health promotion should emphasize practical self-management skills essential for glycemic control and preparation for pregnancy including the use of an appropriate meal plan, selfmonitoring of blood glucose (SMBG), self-administration of insulin and self-adjustment of insulin doses, treatment of hypoglycemia (patient and family members), incorporation of physical activity, and development of stress reduction techniques. Patients should be counseled about fetal and neonatal complications (and possibly long-term health consequences) of maternal diabetes, including the risk of congenital malformations, and the need for effective contraception and optimal glycemic control prior to the next pregnancy. Clinical interventions include dietary control and pharmacological treatment using oral hypoglycemics or insulin. Glycemic control can be self-monitored at home, and glycosylated hemoglobin levels should be evaluated at 1- to 2-month intervals until stable. Follow-up visits should be at 1- to 2-month intervals, and frequent phone contact for adjustment of insulin doses and other aspects of the treatment regimen is advised. Psychosocial interventions should address barriers to adherence with treatment protocol. Referral to and coordination with a dietician and programs such as W.I.C. or Sweet Success in California may enhance treatment efficacy. Stress management should be emphasized because stress can affect dietary control as well as glucose homeostasis.

For women with gestational diabetes in a previous pregnancy, guidelines for internatal care are less well established. The American Diabetes Association recommends that a 75-g oral glucose tolerance test (GTT) be performed 6 to 8 weeks after delivery [41]. The extent to which this recommendation is adhered is presently unknown. We believe that much more can be done in the internatal period to promote maternal health (as well as fetal health in a subsequent pregnancy) among women with gestational diabetes. Because these women are at increased risk for developing type II diabetes, risk assessment should include a fasting glucose once a year. If clinical assessment suggests polycystic ovarian syndrome and/or metabolic syndrome, periodic testing for lipoprotein profiles, fasting glucose, and possibly glucose tolerance test may be warranted [45]. Patients should be evaluated for glucose intolerance before their next pregnancy. For health promotion, all women who have had gestational diabetes should be encouraged to exercise and lose weight if they are obese. Dietary counseling is recommended given their increased risk for future type II diabetes. Presently there are no specific clinical interventions recommended in the internatal period for women with a history of gestational diabetes.

During a subsequent pregnancy, women with pregestational diabetes using oral hypoglycemic agents should be switched to insulin because the safety and efficacy of currently available oral hypoglycemics have not been evaluated for use in pregnancy among women with pregestational diabetes. For women with gestational diabetes in a prior pregnancy, the use of a second-generation oral hypoglycemic agent (i.e. glibenclamide or glyburide) may be considered if gestational diabetes recurs in a subsequent pregnancy. The agent does not cross the placenta and has been shown to be comparable to insulin in improving glucose control for women with gestational diabetes [46]. Weight gain during pregnancy should be closely monitored. 
Women who are underweight, overweight, or obese

A growing number of women of reproductive age in the U.S. are overweight or obese. According to the 1997 National Health Interview Survey, one-third of women ages 18 to 24 and $43 \%$ of women ages 25 to 44 are overweight [47]. The survey also found one in eight women ages 18 to 24 and one in six women ages 25 to 44 are obese [47]. Based on the CDC 2003 Pregnancy Nutrition Surveillance System (PNSS) of nearly 728,000 low-income pregnant women, $43 \%$ had a prepregnancy body mass index (BMI) that is considered overweight or obese, up from less than $30 \%$ in 1989 [48]. Maternal obesity poses a threat to not only subsequent pregnancy outcomes, but also long-term maternal health. Pregnancy itself is a risk factor for maternal obesity. According to the 2003 PNSS, more than $44 \%$ had gained more than the ideal weight gain [48] and, as previously cited [24], weight retention following pregnancy is common. Weight retention needs to be addressed during internatal care, particularly for women who are overweight or obese.

According to the 2003 PNSS, one in eight pregnant women were underweight before pregnancy, and one in four had gained less than the ideal weight gain during pregnancy [48]. Both low pre-pregnancy BMI and poor pregnancy weight gain have been shown to be risk factors for preterm delivery and low birth weight [49].

For women who are underweight, overweight, or obese, an important goal of internatal care is to optimize their weight prior to the next pregnancy. For risk assessment, the BMI can be used because it provides an acceptable approximation of body fat and fair correlation with disease outcomes. Potential causes of underweight (e.g. eating disorders, food insecurity), overweight or obesity (e.g. polycystic ovarian syndrome) need to be evaluated. For health promotion, women who are underweight should be encouraged to gain weight through healthy nutrition; for women who are overweight or obese, clinical guidelines have been established for the identification, evaluation, and treatment of overweight and obesity [50]. These guidelines should be followed for internatal care. The initial goal of weight loss therapy should be to reduce body weight by approximately 10 percent from baseline, and weight loss should be about 1 to 2 pounds per week for a period of about 6 months. This amount of postpartum weight loss in overweight women does not appear to affect the growth of their infants, even if they are exclusively breastfed [51]. Weight loss and weight maintenance therapy should employ the combination of low-calorie diets, increased physical activity, and behavior therapy. Clinical interventions may include weight loss drugs as an adjunct to diet and physical activity for patients with a BMI of $\geq 30$, as well as for patients with a BMI of $\geq 27$ with concomitant risk factors or diseases. After successful weight loss, the likelihood of weight loss maintenance is enhanced by a program consisting of dietary therapy, physical activity, and behavior therapy which should be continued until the next pregnancy. Psychosocial interventions should address barriers to healthy nutrition and coordination with a dietician, WIC and other community programs. The schedule of internatal visits should be individualized; the literature suggests that frequent contacts between professional counselors and patients help promote healthy weight maintenance. In a $s u b$ sequent pregnancy, the Institute of Medicine guidelines for weight gain during pregnancy should be promoted based on prepregnancy BMI [52].

\section{Women who had a prior preterm birth}

Presently this group of women is most often targeted for internatal care. Preterm birth is a leading cause of infant mortality and long-term disabilities in children in the U.S.. Prior preterm birth is the strongest and most consistent predictor of a subsequent preterm birth. This may be due to the fact that many of the biobehavioral risk factors for preterm birth are carried from one pregnancy to the next. The goal of internatal care for these women is to prevent recurrence of preterm birth by addressing these continuing biobehavioral risks prior to their next pregnancy.

\section{Risk assessment}

Women who had a preterm birth should undergo a comprehensive risk assessment, starting with a complete review of their obstetrical history at the two-week visit. Distinction should be made between spontaneous and induced preterm deliveries. Details about the preterm delivery should be obtained. Work-up should be guided by known etiologic pathways (e.g. infectious-inflammatory, vascular, neuroendocrine and pathologic uterine over-distension) [53]. To identify infectious-inflammatory causes, medical records should be reviewed for untreated infections (e.g. asymptomatic bacteriuria), physical examination should be performed to uncover on-going, chronic infections (e.g. periodontal or reproductive tract infections), and placental pathology should be reviewed for evidence of chorioamnionitis [54]. To identify vascular causes, placental pathology should be reviewed for evidence of uteroplacental ischemia and thromboses [54]. The presence of these findings, in the absence of known causes, may indicate work-up for hereditary or acquired thrombophilia [55]. To identify neuroendocrine causes, inquiries should be made about psychosocial stress and support during pregnancy. Special attention should be paid to FINDS (family violence, infections, nutrition, depression, and stress), since these risk factors may be involved in one or more etiologic pathways leading to the occurrence or recurrence of preterm birth. Review of past medical history should focus on conditions that could increase the 
risk of preterm deliveries (e.g. systemic lupus erythematosus or renal disease). Review of family history should identify other preterm deliveries in the family (particularly among first-degree relatives), as well as other conditions associated with preterm deliveries. A thorough review of reproductive history, including other preterm births or pregnancy losses, sexual history including sexually transmitted infections, past and current contraceptive use, and plans for future childbearing and child spacing should be conducted.

\section{Health promotion}

The goal of health promotion is to promote protective factors and reduce risk factors for recurrent preterm births. We will highlight three areas of health promotion: smoking cessation, healthy nutrition, and family planning. First, exposure to cigarette smoking significantly increases the risk of preterm delivery. Smoking cessation (by women and their partners) should be encouraged during internatal care. As previously discussed, effective models of clinical interventions (e.g. 5A's) have been developed [28]. Second, healthy nutrition should be promoted. Low pre-pregnancy BMI has been associated with increased risk for spontaneous preterm birth, while high pre-pregnancy BMI has been associated with increased risk for indicated preterm birth [49]. For women with a history of preterm birth, achieving a healthy weight prior to their next pregnancy may reduce the risk of recurrent preterm birth. A number of programs have demonstrated effectiveness in helping women achieve and maintain healthy weight [56]. Additionally, nutrition may also play an important role in modulating several of the etiologic pathways leading to preterm birth. For example, increased dietary intake of omega-3 polyunsaturated fatty acids may help modulate inflammation [57]. Women should be encouraged to increase consumption of foods rich in omega-3 (the International Society for the Study of Fatty Acids and Lipids recommends that during pregnancy and lactation women must ensure a minimum daily intake of $300 \mathrm{mg}$ of Docosahexaenoic Acid or DHA, an important source of omega-3 fatty acids [58]), and decrease consumption of foods high in saturated fatty acids, trans-fats, and partially-hydrogenated oils. Increasing dietary intake of fruits and vegetables rich in antioxidants and certain phytochemicals should also be encouraged. These recommendations could arguably be adopted on the basis of promoting women's health alone, even in the absence of data supporting their effectiveness in preventing recurrent preterm birth. Third, the importance of family planning cannot be over-emphasized, particularly for women with a history of preterm birth. Both unintended pregnancies and short interpregnancy intervals are associated with increased risk for preterm delivery. Family planning has been shown to reduce unintended pregnancies and promote optimal child spacing. Other BBEEFF topics, such as breast- feeding and back-to-sleep, are also important to address with mothers of preterm babies.

\section{Clinical interventions}

To our knowledge, there has been only one randomized controlled trial for the prevention of recurrent preterm birth in which a specific clinical intervention is initiated during the interpregnancy period [59]. In that study, women with a prior spontaneous preterm birth at less than 34 weeks of gestation were randomized at 3 months postpartum to a course of oral azithromycin and sustained-release metronidazole or placebo. The regimen was repeated every 4 months until conception of another pregnancy. The investigators found no significant difference in the rates of preterm birth between the study and control groups. In fact, women in the treatment group, on average, had lower birth weight babies and delivered 2.4 weeks earlier [59]. In the absence of evidence of effectiveness for preventing recurrent preterm birth, presently no specific clinical intervention can be recommended for women with prior preterm birth in the interpregnancy period.

Several studies have examined the effectiveness of clinical interventions applied to women with prior preterm birth during a subsequent pregnancy for the prevention of recurrent preterm birth. Perhaps the most promising is the use of progesterone. A large randomized controlled trial in the U.S. [60] and two recent meta-analyses [61, 62] found evidence of effectiveness of progestational agents (e.g. 17hydroxyprogesterone caproate) for the prevention of preterm or recurrent preterm birth. However, "because unresolved issues remain, such as optimal route of drug delivery and long-term safety of the drug," the American College of Obstetricians and Gynecologists (ACOG) has issued a committee opinion that "when progesterone is used, it is important to restrict its use only to women with a documented history of a previous spontaneous birth at less than 37 weeks of gestation" [63]. Another promising clinical intervention is the use of fish oil supplementation for the prevention of recurrent preterm birth. In the Fish Oil Trials in Pregnancy (FOTIP) study, fish oil supplementation in a subsequent pregnancy significantly reduced recurrence risk of preterm delivery [64]. There is also emerging evidence that scaling and root planing in pregnant women with periodontitis may reduce preterm birth [65], though no published studies have evaluated the effectiveness of periodontal screening in women with prior preterm birth. Further studies are needed to demonstrate the effectiveness of these interventions.

Perhaps the most controversial clinical intervention is antibiotic treatment of asymptomatic bacterial vaginosis among women with a history of preterm birth [66, 67]. While some studies have shown a benefit, others have not. Efficacy of treatment may depend on the agent used, the route of 
administration, the timing of treatment, test of cure, and a number of host factors. In at least four antibiotic trials in pregnancy involving women with prior preterm birth [59, 65, 68, 69], antibiotic treatment (of bacterial vaginosis, Trichomonas vaginalis, or periodontal infection) was associated with higher, not lower, incidence of recurrent preterm birth. Clearly there is a need for further intervention studies, guided by better understanding of pharmacokinetics and pharmacogenetics, disease mechanisms and host response, as this remains a potentially important area of intervention for the prevention of recurrent preterm birth.

\section{Psychosocial interventions}

A growing body of evidence suggests that maternal psychosocial stress is an important risk factor for preterm delivery, and that risk can be mitigated by reducing stress and/or increasing support. However, most extant studies of social support during pregnancy suffer from methodological flaws [70], and there is a paucity of research on the effectiveness of preconception or internatal support. The provision of social, clinical and parenting support during the internatal period, as previously described, may be quite valuable to mothers of a preterm infant, who often has special healthcare and other developmental needs that require additional support services. Such services may also be particularly beneficial to low-SES mothers, who may face greater burdens with less personal and social resources to deal with them. More research is needed to identify effective models of internatal psychosocial interventions for women with a history of preterm delivery.

\section{Service coordination and integration}

Women who have had a preterm birth may require multiple services during the internatal period, particularly if their children have significant disabilities and special healthcare needs. Providing integrated services, including linkages to early intervention or other early child development programs for preterm or LBW infants, should be a primary objective of care for these women. Several models of care coordination and service integration have been developed [71, 72]. One of the most successful models is the Olds nurse home visitation program [73]. The program provided nurse home visitation during pregnancy and the first two years postpartum. Evaluation of the Memphis/Shelby County program [73] found women who received home visits by nurses had fewer closely spaced subsequent pregnancies, longer internatal intervals, and fewer months of using public assistance programs, compared to a control group of women. There were, however, no statistically significant program effects on LBW or stillbirth in the subsequent pregnancy. The program is technically not an "internatal care" program and not targeted specifically to women who had a preterm birth, but this model could potentially be adapted by Healthy Start or other public health or community-based programs to provide service coordination and integration for internatal care using community nurses. We recommend that all women with prior preterm birth be offered care coordination and/or home visitation during internatal care.

\section{Schedule of visits}

The schedule of internatal visits for women with prior preterm birth needs to be individualized (with a minimum of three visits plus annual visits, as previously described), depending on the clinical and psychosocial needs of the woman and family. During their subsequent pregnancy, these women should be supervised by a maternal fetal medicine specialist, either directly or indirectly through consultations.

In sum, given the large impact of preterm birth on infant mortality and childhood disabilities and the high rates of recurrence in a subsequent pregnancy, women with prior preterm birth could be one of the most critical target groups for enhanced internatal care. Other high-risk women (e.g. women with prior fetal death), could also benefit from such program; we are unable to describe the content of their care in this paper due to space limitation and will do so in a series of papers to follow. The goal of such program is to prevent recurrence of preterm birth by addressing known biobehavioral pathways (e.g. infections/inflammation, neuroendocrine, vascular, etc) prior to the next pregnancy. There is a great need for more comprehensive and systematic risk assessment and health promotion during internatal care, as well as more basic, clinical and intervention research to identify effective clinical and psychosocial interventions for the prevention of recurrent preterm birth.

\section{Conclusion}

In this paper, we began to define the contents of internatal care. We recommended expanding the current six-week postpartum visit to three or more internatal visits (at two weeks, six weeks, and six months postpartum, and annual visits beginning at one-year postpartum). We suggested some core contents that all women should receive during internatal care, including risk assessment (e.g. FINDS), health promotion (e.g. BBEEFF), clinical and psychosocial interventions. For women with chronic health conditions such as hypertension, diabetes, or weight problems, we identified clinical guidelines for their evaluation, treatment, and follow-up during the internatal period. For women who had a preterm birth, we proposed an internatal care model based on known etiologic pathways, with the goal of preventing recurrence by addressing these biobehavioral pathways prior to the next 
pregnancy. We suggested enhancing service integration for women and families, including possibly care coordination or home visitation for women with prior preterm birth.

We were limited in our effort to define the contents of internatal care by the paucity of research on the internatal period. Many pre-disease pathways leading to recurrence of adverse birth outcomes have not yet been elucidated. More importantly, even less is known about the trajectories to longterm women's health or child health, or how to alter these pathways and trajectories. There is a need for more intervention studies evaluating the effectiveness of the various components of internatal care. More importantly, we need more research on mechanisms of disease to guide the design of internatal interventions. Our proposal will need to be updated and revised continually as new research data emerge.

We were also limited by the paucity of research on community-level or public health interventions during the internatal period. While our paper focused on the contents of the clinical visits, we believe that provision of internatal care should not be limited to the clinician's office. For example, clinical interventions to control hypertension may be more effective if they are supported by community-level or public health interventions, such as implementation of the American Public Health Association resolution that the food manufacturers and restaurants reduce sodium in the food supply by 50 percent over the next decade [37]. The 5A's of smoking cessation may be more effective if they are supported by multilevel interventions at the interpersonal (e.g. targetting partner smoking), community and institutional (e.g. billboards and marketing of tobacco products, access to smoking cessation programs), and policy levels (e.g. ban on smoking in public space, promotion of smoke-free environments, and increased taxation on tobacco products) [3]. We believe that the ultimate success of internatal care may hinge on the development of a much broader and more integrated conception of internatal care than currently prevails with prenatal care [2].

Presently there are numerous public health programs offering some components of internatal care, including many Healthy Start programs. They could provide the ideal social laboratory settings for implementing and evaluating various community-level interventions. While there are significant challenges to this type of research, it needs to be done. Identifying what internatal interventions work in the community may be just as important as identifying what works in the clinical setting. Such research needs to be guided by the principles and methods of community-based participatory research (CBPR) [74] and be held to scientifically rigorous standards.

Although more research is needed to identify effective internatal interventions, we believe we cannot wait for the perfect studies to be done. We must begin to act. Most of our recommendations can be justified on the ba- sis of providing good preventive and primary care for women and children, even in the absence of conclusive evidence supporting their effectiveness in improving subsequent pregnancy outcomes. For the most part they reflect current practice standards recommended by major national organizations (Table 1); most of these are considered standards of care or best practices in women's and children's health care. The only recommendations without the endorsement of a national organization-screening for psychosocial stress and provision of psychosocial support-are based on our collective opinions; we strongly believe these are important to reduce allostatic load on women's health and improve subsequent pregnancy outcome. In this paper we did not attempt to address all the institutional components and challenges in developing a successful model of internatal care, only the content of such a program. This paper reflects our effort to start the process of defining the contents of internatal care. We hope that this paper can be used to stimulate further dialogues about internatal care. Such dialogues, we believe, are needed to move forward research, practice, and policy on internatal care, and to move this nation one step closer toward the provision of a more universal, longitudinally-integrated continuum of women's healthcare.

Acknowledgments Support for this work was provided in part by the Los Angeles Best Babies Collaborative, a program of First 5 LA; the Maternal and Child Health Bureau Leadership Training Program in Schools of Public Health; and the Community Child Health Network funded jointly by the National Institute of Child Health and Development and the National Institute of Nursing Research. We also acknowledge Drs. Karla Damus, Jamie McGregor, and Ronald Gibbs for their advice and guidance in the development of the manuscript.

\section{References}

1. Lu MC, Tache V, Alexander G, Kotelchuck M, Halfon N. Preventing LBW: is prenatal care the answer? J Matern Fetal Neonat Med 2003;13:362-80.

2. Alexander GR, Kotelchuck M. Assessing the role and effectiveness of prenatal care: History, challenges, and directions for future research. Public Health Reports 2001;116:306-16.

3. Lu MC, Halfon N. Racial and ethnic disparities in birth outcomes: A life-course perspective. Maternal and Child Health Journal 2003;7:13-30.

4. Johnson K, Posner SF, Biermann J, Cordero JF, Atrash HK, Parker CS, et al. CDC/ATSDR Preconception Care Work Group; Select Panel on Preconception Care.Recommendations to improve preconception health and health care-United States. A report of the CDC/ATSDR Preconception Care Work Group and the Select Panel on Preconception Care. MMWR Recomm Rep. 2006 Apr 21;55(RR-6):1-23.

5. The Interconception Health Promotion Initiative Team. Interconception Health Promotion Initiative final report. Denver:Colorado Trust. 2003. Access at: http://www.coloradotrust. org/repository/publications/pdfs/IHPIFinalReport04.pdf.

6. Dunlop A, Brann A. African American women at Grady Memorial Hospital (GMH). Atlanta, GA: Presented at the National Summit on Preconception Care; June 21, 2005. 
7. Culhane J. Philadelphia Collaborative Preterm Prevention Project. Access at: http://www.dsf.health.state.pa.us/health/CWP/ view.asp? A $=175 \& Q$ QUESTION_ID $=238556$.

8. Adams MM, Elam-Evans LD, Wilson HG, Gilbertz DA. Rates of and factors associated with recurrence of preterm delivery. JAMA. 2000;283:1591-6.

9. Expert Panel on the Content of Prenatal Care. Caring for our future: The content of prenatal care. Washington, DC: Public Health Service; 1989.

10. Gazmararian JA, Lazorick S, Spitz AM, et al. Prevalence of violence against pregnant women. JAMA 1996;275(24):1915-20.

11. Martin SL, Mackie L, Kupper LL, Buescher PA, Moracco KE. Physical abuse of women before, during, and after pregnancy. JAMA. 2001;285:1581-4.

12. Harrykissoon SD, Rickert VI, Wiemann CW. Prevalence and patterns of intimate partner violence among adolescent mothers during the postpartum period. Arch Pediatr Adolesc Med. 2002;156:325-30.

13. Nelson HD, Nygren P, McInerney Y, Klein J. Screening Women and Elderly Adults for Family and Intimate Partner Violence: A Review of the Evidence for the U.S. Preventive Services Task Force. Ann Intern Med 2004;140:387-96.

14. Scannapieco FA, Bush RB, Paju S. Periodontal disease as a risk factor for adverse pregnancy outcomes. A systematic review. Ann Periodontol. 2003;8:70-8.

15. Scannapieco FA, Bush RB, Paju S. Associations between periodontal disease and risk for atherosclerosis, cardiovascular disease, and stroke. A systematic review. Ann Periodontol. 2003;8:3853.

16. U.S. Preventive Services Task Force. Screening for Chlamydial Infection: Recommendations and Rationale. Am J Prev Med 2001;20(3S):90-4.

17. Harrison GG, DiSogra CA, Manalo-LeClair G, Aguayo J, Yen W. Over 2.2 Million Low-Income California Adults are Food Insecure; 658,000 Suffer Hunger. Los Angeles, CA: UCLA Center for Health Policy Research; 2002.

18. Gaynes BN, Gavin N, Meltzer-Brody S, Lohr KN, Swinson T, Gartlehner G, Brody S, Miller WC. Perinatal Depression: Prevalence, Screening Accuracy, and Screening Outcomes. Washington, DC: Agency for Healthcare Research and Quality. Evidence Report/Technology Assessment: Number 119. AHRQ Pub. No. 05-E006-1. February 2005.

19. McEwen BS. Protective and damaging effects of stress mediators. N Eng J Med. 1998;338:171-9.

20. Wadhwa PD, Sandman CA, Garite TJ. The neurobiology of stress in human pregnancy: implications for prematurity and development of the fetal central nervous system. Prog Brain Res. 2001;133:131-42.

21. American Academy of Pediatrics Work Group on Breastfeeding. Breastfeeding and the use of human milk. Pediatrics 1997;100:1035-7.

22. Lu MC, Lange L, Slusser W, Hamilton J, Halfon N. Provider encouragement of breast-feeding: evidence from a national survey. Obstet Gynecol. 2001;97:290-5.

23. Campbell Daily K. Update on sudden infant death syndrome. Current Opinion in Pediatrics. 2004;16:227-232.

24. Keppel KG, Taffel SM. Pregnancy-related weight gain and retention: implications of the 1990 Institute of Medicine guidelines. Am J Public Health. 1993;83:1100-3.

25. National Heart, Lung, and Blood Institute. Clinical Guidelines on the Identification, Evaluation, and Treatment of Overweight and Obesity in Adults. Washington, DC: National Heart, Lung, and Blood Institute. NIH Publication No. 98-4083. 1998.

26. Martin JA, Hamilton BE, Sutton PD, Ventura SJ, Menacker F, Munson ML. Births: Final data for 2003. Natl Vital Stat Rep. 2005 Sep 8;54(2):1-116.
27. Lumley J, Oliver SS, Chamberlain C, Oakley L. Interventions for promoting smoking cessation during pregnancy. Cochrane Database Syst Rev. 2004 Oct 18;(4):CD001055.

28. U.S. Department of Health and Human Services, Public Health Service. Fiore MC, Bailey WC, Cohen SJ, et al. Treating tobacco use and dependence. Clinical practice guideline. Rockville (MD): U.S. Department of Health and Human Services, Public Health Service; 2000.

29. Centers for Disease Control and Prevention. Alcohol use among women of childbearing age-United States, 1991-1999. MMWR Morb Mortal Wkly Rep. 2002;51:273-6.

30. American College of Obstetricians and Gynecologists. Substance Abuse in Pregnancy. Substance abuse in pregnancy. ACOG Technical Bulletin Number 195. Int J Gynaecol Obstet. 1994;47:73-80.

31. Brown SS, Eisenberg L, editors. The best intentions: Unintended pregnancy and the well-being of children and families. Washington, DC: National Academy of Sciences; 1995.

32. Zhu BP. Effect of interpregnancy interval on birth outcomes: findings from three recent US studies. Int $\mathrm{J}$ Gynaecol Obstet. 2005;89(Suppl 1):S25-33.

33. Lumley J, Watson L, Watson M, Bower C. Periconceptional supplementation with folate and/or multivitamins for preventing neural tube defects. Cochrane Database Syst Rev. 2001;(3):CD001056.

34. Centers for Disease Control and Prevention. Recommendations for the use of folic acid to reduce the number of cases of spina bifida and other neural tube defects. MMWR 1992;41(no. RR-14).

35. Grady MA, Bloom KC. Pregnancy outcomes of adolescents enrolled in a Centering Pregnancy program. J Midwifery Womens Health. 2004;49:412-20.

36. American College of Obstetricians and Gynecologists. ACOG technical bulletin. Preconception care. Number 205-May 1995. American College of Obstetricians and Gynecologists. Int $\mathrm{J}$ Gynaecol Obstet. 1995 Aug;50(2):201-7.

37. Chobanian AV, Bakris GL, Black HR, Cushman WC, Green LA, Izzo JL Jr, Jones DW, Materson BJ, Oparil S, Wright JT Jr, Roccella EJ. Seventh report of the Joint National Committee on Prevention, Detection, Evaluation, and Treatment of High Blood Pressure. Hypertension 2003;42:1206-52.

38. National Heart, Lung, and Blood Institute. National High Blood Pressure Education Program Working Group Report on High Blood Pressure in Pregnancy. Bethesda (MD): National High Blood Pressure Education Program, National Heart, Lung, and Blood Institute, National Institutes of Health, 2000.

39. Smith GC, Pell JP, Walsh D. Pregnancy complications and maternal risk of ischaemic heart disease: a retrospective cohort study of 129,290 births. Lancet. 2001 Jun 23;357(9273):2002-6.

40. Korenbrot CC, Steinberg A, Bender C, Newberry S. Preconception care: a systematic review. Matern Child Health J. 2002;6:75-88.

41. American Diabetes Association. Gestational diabetes mellitus. Diabetes Care 2004;27(Suppl 1):S88-90.

42. Kim C, Newton KM, Knopp RH. Gestational diabetes and the incidence of type 2 diabetes: a systematic review. Diabetes Care 2002;25:1862-8.

43. Aerts L, Van Assche FA. Intra-uterine transmission of disease. Placenta. 2003;24:905-11.

44. American Diabetes Association. Preconception care of women with diabetes. Diabetes Care 2004;27(Suppl 1):S76-8.

45. The Rotterdam ESHRE/ASRM-Sponsored PCOS Consensus Workshop Group. Revised 2003 consensus on diagnostic criteria and long-term health risks related to polycystic ovary syndrome. Fertil Steril 2004;81:19-25.

46. Langer O. When diet fails: insulin and oral hypoglycemic agents as alternatives for the management of gestational diabetes mellitus. J Matern Fetal Neonatal Med. 2002;11:218-25.

47. http://www.cdc.gov/nchs/products/pubs/pubd/hestats/3and4/ overweight.htm. 
48. http://www.cdc.gov/pednss/pnss_tables/tables_health_indicators.htm.

49. Hendler I, Goldenberg RL, Mercer BM, Iams JD, Meis PJ, Moawad AH, et al. The Preterm Prediction Study: association between maternal body mass index and spontaneous and indicated preterm birth. Am J Obstet Gynecol. 2005;192:882-6.

50. National Heart, Lung, and Blood Institute. Clinical Guidelines on the Identification, Evaluation, and Treatment of Overweight and Obesity in Adults. Washington, DC: National Heart, Lung, and Blood Institute, National Institutes of Health, 2000. NIH Publication No. 98-4083. 1998.

51. Lovelady CA, Garner KE, Moreno KL, Williams JP. The effect of weight loss in overweight, lactating women on the growth of their infants. NEJM 2000;342:448-53.

52. Institute of Medicine Committee on Nutritional Status During Pregnancy and Lactation, Food and Nutrition Board. Nutrition During Pregnancy Part I: Weight Gain. Washington DC: National Academy Press; 1990.

53. Lockwood CJ. Testing for risk of preterm delivery. Clin Lab Med. 2003;23:345-60.

54. Salafia CM. Recurrent obstetric complications: how placental pathology can contribute to cost-effective clinical evaluation and a rational clinical care plan. Curr Womens Health Rep. 2002;2:65-71.

55. Lockwood CJ. Inherited thrombophilias in pregnant patients: detection and treatment paradigm. Obstet Gynecol. 2002;99:333-41.

56. Tsai AG, Wadden TA. Systematic review: an evaluation of major commercial weight loss programs in the United States. Ann Intern Med. 2005;142:56-66.

57. McGregor JA, Allen KG, Harris MA, Reece M, Wheeler M, French JI, Morrison J. The omega-3 story: nutritional prevention of preterm birth and other adverse pregnancy outcomes. Obstet Gynecol Surv. 2001;56(5 Suppl 1):S1-13.

58. International Society for the Study of Fatty Acids and Lipids. Workshop on the Essentiality of and Recommended Dietary Intakes for Omega-6 and Omega-3 Fatty Acids. April 7-9, 1999. http://www.issfal.org.uk/Welcome/AdequateIntakes.asp.

59. Andrews W, Goldenberg R, Hauth J, Cliver S. Interconceptional antibiotics to prevent spontaneous preterm birth (SPTB): a randomized trial. Abstract presented at the 24th Annual meeting of Society for Maternal-Fetal Medicine (SMFM) held in New Orleans, LA February 2004.

60. Meis PJ, Klebanoff M, Thom E, Dombrowski MP, Sibai B, Moawad AH et al. for the National Institute of Child Health and Human Development Maternal-Fetal Medicine Units Network. Prevention of recurrent preterm delivery by 17 alphahydroxyprogesterone caproate. N Engl J Med. 2003;348:2379-85.

61. Sanchez-Ramos L, Kaunitz AM, Delke I. Progestational agents to prevent preterm birth: a meta-analysis of randomized controlled trials. Obstet Gynecol. 2005;105:273-9.

62. Dodd JM, Crowther CA, Cincotta R, Flenady V, Robinson JS. Progesterone supplementation for preventing preterm birth: a systematic review and meta-analysis. Acta Obstet Gynecol Scand. 2005;84:526-33.

63. American College of Obstetricians and Gynecologists. Use of progesterone to reduce preterm birth. Int J Gynaecol Obstet. 2004;84:93-4.

64. Olsen SF, Secher NJ, Tabor A, Weber T, Walker JJ, Gluud C. Randomised clinical trials of fish oil supplementation in high risk pregnancies. Fish Oil Trials In Pregnancy (FOTIP) Team. BJOG. 2000;107:382-95.

65. Jeffcoat MK, Hauth JC, Geurs NC, Reddy MS, Cliver SP, Hodgkins PM, Goldenberg RL. Periodontal disease and preterm birth: results of a pilot intervention study. J Periodontol. 2003;74:1214-8.

66. Okun N, Gronau KA, Hannah ME. Antibiotics for bacterial vaginosis or Trichomonas vaginalis in pregnancy: a systematic review. Obstet Gynecol. 2005;105:857-68.

67. McDonald H, Brocklehurst P, Parsons J. Antibiotics for treating bacterial vaginosis in pregnancy. Cochrane Database Syst Rev. 2005 Jan 25;(1):CD000262.

68. Klebanoff MA, Carey JC, Hauth JC, Hillier SL, Nugent RP, Thom EA, et al. for the National Institute of Child Health and Human Development Network of Maternal-Fetal Medicine Units. Failure of metronidazole to prevent preterm delivery among pregnant women with asymptomatic Trichomonas vaginalis infection. $\mathrm{N}$ Engl J Med. 2001;345:487-93.

69. Andrews WW, Sibai BM, Thom EA, Dudley D, Ernest JM, McNellis D, et al. for the National Institute of Child Health \& Human Development Maternal-Fetal Medicine Units Network. Randomized clinical trial of metronidazole plus erythromycin to prevent spontaneous preterm delivery in fetal fibronectin-positive women. Obstet Gynecol. 2003;101:847-55.

70. Lu Q, Lu MC, Schetter CD. Learning from success and failure in psychosocial intervention: an evaluation of low birth weight prevention trials. J Health Psychol. 2005;10:185-95.

71. Willis WO, Eder CH, Lindsay SP, Chavez G, Shelton ST. Lower rates of low birthweight and preterm births in the California Black Infant Health Program. J Natl Med Assoc. 2004 Mar;96(3):315-24.

72. McCormick MC, Deal LW, Devaney BL, Chu D, Moreno L, Raykovich KT. The impact on clients of a community-based infant mortality reduction program: the National Healthy Start Program Survey of Postpartum Women. Am J Public Health. 2001;91:1975-7.

73. Kitzman H, Olds DL, Sidora K, Henderson CR Jr, Hanks C, Cole $\mathrm{R}$, et al. Enduring effects of nurse home visitation on maternal life course: a 3-year follow-up of a randomized trial. JAMA. 2000;283:1983-9.

74. O'Fallon L, Tyson F, Dearry A, editors. Successful Models of Community-Based Participatory Research (2000)., National Institute of Environmental Health Sciences: Research Triangle Park, NC. Available at: http://www.niehs.nih.gov/translat/cbr-final.pdf. Accessed February 3, 2005. 